\title{
BMJ Open Open-label placebo for insomnia (OPIN): study protocol for a cohort multiple randomised controlled trial
}

\author{
Ben Colagiuri (D) , ${ }^{1}$ Louise Sharpe, ${ }^{1}$ Zahava Ambarchi, ${ }^{1}$ Nick Glozier, ${ }^{2}$ \\ Delwyn Bartlett, ${ }^{3}$ Daniel S J Costa, ${ }^{1}$ Amelia Scott ${ }^{1}$
}

To cite: Colagiuri B, Sharpe L, Ambarchi Z, et al. Openlabel placebo for insomnia (OPIN): study protocol for a cohort multiple randomised controlled trial. BMJ Open 2021;11:e044045. doi:10.1136/ bmjopen-2020-044045

- Prepublication history and additional materials for this paper are available online. To view these files, please visit the journal online (http://dx.doi. org/10.1136/bmjopen-2020044045).

Received 23 August 2020

Revised 29 January 2021

Accepted 03 February 2021

Check for updates

(C) Author(s) (or their employer(s)) 2021. Re-use permitted under CC BY-NC. No commercial re-use. See rights and permissions. Published by BMJ.

${ }^{1}$ School of Psychology, The University of Sydney, Sydney, New South Wales, Australia ${ }^{2}$ Brain and Mind Centre, The University of Sydney, Sydney, New South Wales, Australia ${ }^{3}$ Woolcock Institute, The University of Sydney, Sydney, New South Wales, Australia

Correspondence to

Dr Ben Colagiuri;

ben.colagiuri@sydney.edu.au

\section{ABSTRACT}

Introduction Insomnia is a prevalent sleep disorder that causes substantial personal and societal harm. There is evidence that placebo interventions can reduce insomnia symptoms, but this research has involved deceptively administering the placebo under the guise of a real medication (conventional placebo, CP), which has obvious ethical constraints. Open-label placebo (OLP) treatment, in which a placebo is administered with full disclosure that there are no active ingredients, has been proposed as a method of using the placebo effect ethically, but the efficacy and acceptability of OLP for insomnia is currently unknown.

Methods and analysis This study uses a cohort multiple randomised controlled trial design to compare OLP, CP and no treatment for insomnia. Two-hundred and sixtyseven participants with self-reported insomnia symptoms (Insomnia Severity Index, ISI $\geq 10$ ) will be recruited into an observational study and have their sleep monitored over a 2-week period. Participants will then be randomised to one of three groups: invite to OLP, invite to CP described deceptively as a new pharmacological agent, or no invite/ observational control. Those in OLP and CP accepting the invite receive identical placebos for a 2-week treatment period while sleep is monitored in all participants. The primary outcome is ISI at the end of the treatment period. Secondary outcomes include treatment uptake and clinically significant response rates, objective and subjective sleep parameters, fatigue, mood, expectancy, treatment satisfaction and side effects. Predictors of uptake and responses to OLP and CP will be explored. Ethics and dissemination The trial has been approved by The University of Sydney Human Research Ethics Committee. Written informed consent is obtained from every participant. OLP and CP participants accepting the invite undergo an additional consent process. Results will be disseminated via peer-reviewed conference proceedings and publications.

Trial registration number ACTRN12620001080910.

\section{INTRODUCTION}

Insomnia is the most common sleep disorder, with an estimated diagnostic prevalence of $10 \%^{1-3}$ and symptom prevalence of $30 \%^{34}$ in adults. Higher prevalence rates have been reported in medical settings, ranging from $20 \%$ to $56 \%,{ }^{2-6}$ with up to $90 \%$ of patients

\section{Strengths and limitations of this study}

This will be the first study to test whether openlabel placebo (OLP) is effective and acceptable for insomnia.

- The use of a cohort multiple randomised controlled trial design provides a more ecologically valid no treatment comparison and will allow us to compare the efficacy and uptake of OLP relative to conventional placebo.

- The inclusion of actigraphy means that we can assess the effect of OLP on both self-report and objective sleep outcomes.

- Predictors of uptake and any resulting placebo effect will be explored, including expectancy and baseline insomnia severity.

- Because of the nature of the study, participants and researchers cannot be blind to treatment allocation, but the data analysis will be conducted by a blind researcher.

being prescribed pharmacotherapy. ${ }^{7} \quad 8$ Insomnia is characterised by difficulty initiating or maintaining sleep along with daytime impairment and/or distress and carries substantial personal and socioeconomic burden. ${ }^{9} 10$ People with insomnia have poorer quality of life, greater risk of experiencing other medical conditions and psychiatric disorders, increased healthcare utilisation and reduced work productivity. ${ }^{11-13}$ While a range of pharmacological treatments are available for insomnia, those that demonstrate efficacy are associated with significant risks in terms of adverse effects and the potential for dependence (eg, benzodiazepines), ${ }^{14}$ whereas those with lower risk profiles have limited efficacy (eg, melatonin). ${ }^{15}$ Cognitivebehavioural therapy for insomnia (CBT-I) has been recommended as first line treatment for insomnia, ${ }^{2}{ }^{3}$ however, CBT-I is not always accessible ${ }^{3}$ and both practitioners and people with insomnia appear more willing to persist with pharmacological rather than psychological interventions. ${ }^{8} 1617$ As such, 
there remains a pressing need to identify safe and effective treatments to combat the burden of insomnia.

Interestingly, many randomised placebo-controlled trials of insomnia interventions find that participants allocated to placebo treatment experience significant improvement. ${ }^{18-20}$ This suggests that insomnia may be responsive to the placebo effect, whereby the treatment context itself elicits positive expectancies that trigger improvement. ${ }^{21-23}$ Importantly, improvement in placebo groups of these trials holds even when directly compared with no treatment, ${ }^{24}$ indicating that placebo treatment generates more improvement in insomnia than can be accounted for by other factors, such as, spontaneous recovery and regression to the mean. ${ }^{25}$ Therefore, it may be possible to harness the placebo effect to reduce the burden of insomnia.

Placebo interventions likely carry fewer adverse events than pharmacological interventions and have lower cost than psychological interventions. ${ }^{26}$ On the other hand, the deception typically associated with placebo administration presents a significant barrier to its clinical use because of the violation of patient trust and informed consent. ${ }^{27}$ However, this barrier is based on the assumption that deception is necessary to elicit a placebo effect, which has recently been called into question by 'openlabel placebo' (OLP) trials. ${ }^{28}$

OLP trials involve administering placebo treatment with full disclosure that the treatment is in fact a placebo, meaning there is no deception. Only a handful of randomised controlled trials (RCTs) testing OLP have been conducted to date and none with insomnia, but the available data suggest some promising results. For example, in an RCT comparing 3 weeks of OLP with 'treatment as usual' (TAU) for chronic pain, Carvalho et $a l^{29}$ found that OLP significantly reduced pain and disability, with moderate to large effect sizes. Similar results have been found in RCTs of OLP for irritable bowel syndrome ${ }^{30}$ depression ${ }^{31}$ and allergic rhinovirus. ${ }^{32}$ As a result, there have been increasing calls to explore the potential efficacy of OLP in clinical practice. ${ }^{28} 33$

Despite the promising preliminary findings, several criticisms of existing OLP trials have been raised. The most common criticism concerns the types of control group used, typically TAU or waitlist control. The very nature of OLP treatment means that participants and researchers are not blind to treatment allocation, potentially introducing problems with demand characteristics and experimenter bias. ${ }^{28}$ While that may be difficult to avoid, a further problem is that knowingly being allocated to receive no treatment may induce nocebo effects and thereby poorer outcomes in the control group, artificially inflating the apparent efficacy of OLP treatment. ${ }^{28}{ }^{34}$ In addition to concerns regarding the type of control groups used, a second potential important limitation is that participants in OLP trials are usually recruited via advertisements explicitly describing the intervention as a "novel mind-body treatment'. ${ }^{290}$ Little is known about the characteristics of individuals who volunteer to participate in 'novel mind-body treatment' research, but differences between such samples and the general population could significantly limit the generalisability of existing OLP trials. If only those who already hold strong beliefs about the power of the mind are enrolled, then this could overestimate the efficacy of OLP effects in the general population. A final limitation is that existing OLP trials have failed to include a comparison with conventional (deceptive) placebo (CP) treatment, which is important to evaluate the relative cost-benefit of OLP versus CP.

To address this, the current study tests the efficacy of OLP for insomnia (OPIN) using a novel cohort multiple RCT (cmRCT) design comparing OLP, CP and no treatment. The cmRCT involves a two-stage consent process whereby participants are first recruited to an observational study (with no mention of intervention) and are then randomised to be invited to the treatment arms or to remain in the observational arm (ie, act as controls). ${ }^{35}$ This design allows us to compare the efficacy and uptake of OLP versus CP, relative to a no treatment group, in a more generalisable sample of participants not specifically interested in mind-body treatments, and in a scenario whereby participants in the control group are unaware they are missing out on a potentially desirable treatment. The protocol and study design are guided by the recommendations set out in the Standard Protocol Items: Recommendations for Interventional Trials (SPIRIT) 2013 Statement. ${ }^{36}$ The results will provide first ever evidence concerning whether OLP is an effective treatment for insomnia and the strongest test of OLP effects in general to date.

\section{Objectives}

Primary objective

1. Determine whether OLP is associated with reductions in self-report insomnia symptoms, measured by the Insomnia Severity Index (ISI), compared with CP and no treatment.

\section{Secondary objectives}

1. Determine whether OLP is associated with improvements in objective and subjective sleep parameters, daytime fatigue, depression, anxiety, stress, expectancy, treatment satisfaction and side effects, relative to $\mathrm{CP}$ and no treatment.

2. Determine whether OLP is associated with clinically significant improvements in insomnia (response rate), relative to $\mathrm{CP}$ and no treatment.

3. Determine the rate of uptake of OLP relative to CP.

4. Identify which demographic, individual, and clinical characteristics predict uptake and the placebo effect (as measured on ISI scores, number of respondents etc.) following OLP and CP.

\section{METHOD AND ANALYSIS}

\section{Trial design}

As shown in figure 1, the OPIN trial will use a parallel three-arm cmRCT design ${ }^{35}$ comparing OLP, CP and no 


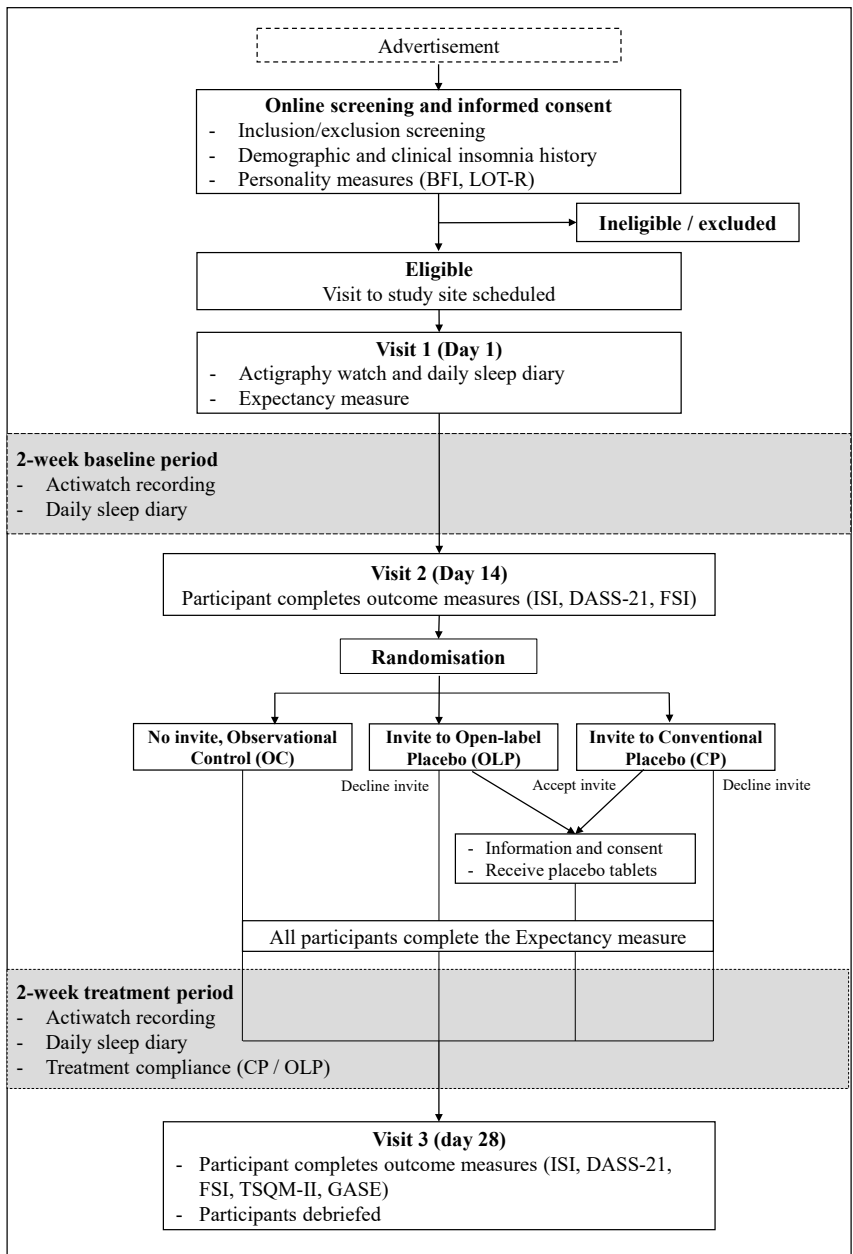

Figure 1 Trial design and flow chart. BFI, Big-Five Inventory; DASS-21, Depression Anxiety Stress Scales; FSI, Fatigue Symptom Inventory; GASE, Generic Assessment of Side Effects; ISI, Insomnia Severity Index; LOT-R, Life Orientation Test-Revised; TSQM-II, Treatment Satisfaction Questionnaire for Medication-Version II.

treatment/observational control (OC) for insomnia. In the first stage, a cohort of participants with self-reported insomnia symptoms will be recruited into a 2-week observational (baseline) period. In the second stage, participants will be randomised to one of three groups: invite to OLP, invite to CP or no invite/OC. OLP will be openly described as consisting of no active ingredient and instead aiming to capitalise on the placebo effect. CP will be described as a new pharmacological agent designed to promote sleep. Participants consenting to OLP or CP will be administered placebo medication, while those allocated to $\mathrm{OC}$ will continue to be observed for the 2-week treatment period.

The study Steering Committee (principal investigator (PI), associate investigators, study coordinator and statistician) will meet every 6 months to review the study, ensuring adherence to all ethical, regulatory and clinical trial guidelines. If higher-than-anticipated attrition rates occur, the Steering Committee will investigate whether the sample size needs to be increased to maintain power, and if so, will seek the appropriate modifications. A Data Monitoring Committee will not be implemented because all participants receive placebos and adverse events are anticipated to be low. Although early study termination is unanticipated, if deemed necessary, only the PI will have the authority to terminate the study.

\section{Participants}

To be eligible, participants must report an ISI $\geq 10$, be at least 18 years old, be proficient in English and be able to attend the study site three times over 1 month. The following exclusion criteria will apply: (1) sleep disorder other than insomnia, (2) currently pregnant, planning to conceive in the next 3 months, breast feeding or $<1$ year post partum, (3) serious medical illness requiring invasive treatment/surgery (eg, cancer) or heavy substance use, (4) severe psychiatric comorbidity (eg, psychosis, bipolar disorder, depression) or risk of self-harm or suicidality, (5) currently receiving psychological treatment or taking regular (ie, $\geq 1 /$ week) medication for sleep (including prescription or over-the-counter medications, herbal supplements, homeopathic preparations), (6) undertaking shift work (fixed or rotating, including regular night shifts), and/or (7) intending to travel to a destination $>2$ hours' time difference in the next 3 months. An ISI score of $\geq 10$ was chosen because it has been suggested to indicate clinically significant insomnia, ${ }^{37}$ with high sensitivity and specificity in community samples,${ }^{38}$ and is frequently used in RCTs of sleep interventions. ${ }^{39}{ }^{40}$ Participants will be reimbursed $\$ A 60$ upon completion of the study and will be provided with 12 months free access to Sleepio ${ }^{\mathrm{TM}}$, a commercially available digital CBT app found to reduce insomnia symptoms. ${ }^{41}$

\section{Study setting}

The study will take place at The University of Sydney, Australia. The study will be advertised online (eg, university research volunteer sites, Facebook) with a link to the study website. The study website includes information about the observational component of the study, researcher contact details, the information sheet and consent form, and a link to complete the online screening measures. Eligible and consenting participants will be contacted and invited to attend the study site to commence participation.

\section{Materials and measures}

\section{Placebo capsules}

Participants in the OLP and CP arms will receive a bottle containing identical 28 blue and white plant-based capsules containing microcrystalline cellulose. Bottles for the OLP and CP treatment arms will be labelled 'Openlabel Placebo Capsules' and '(Codename) Capsules' (the codename is a 7-digit alphanumeric sequence that will be the same for all participants allocated to CP, however, is omitted here to avoid the protocol appearing in any internet searches participants may undertake), respectively. 


\section{Primary outcome}

ISI. ${ }^{37}$ The ISI is a brief, validated 7-item self-report questionnaire assessing insomnia symptomatology on a 5-point scale. Items addressed include the severity of sleep onset, maintenance and early awakening difficulties in the last 2 weeks and are rated from $0=$ 'none' to $4=$ "very severe'. Other items rate sleep dissatisfaction, distress, interference and noticeability to others. Scores are summed to obtain a total score from 0 to 28 , with the following clinical cut-offs: no clinically significant insomnia (0-7), subthreshold insomnia (8-14), moderate insomnia (15-21) and severe insomnia (22-28). The ISI is a reliable and valid measure in clinical and research settings, with sound internal consistency coefficients (0.74-0.78), and moderate concurrent validity $(0.32-0.91)$ between the ISI and daily sleep diary. ${ }^{37}$

\section{Secondary outcomes}

Uptake of OLP and CP. Uptake of OLP and CP will be measured simply as the proportion of participants accepting the invite to each treatment arm.

Response rate. Clinically significant improvements in insomnia will be defined as the rate of participants obtaining a 6-point or greater reduction on the ISI from baseline to post-treatment ${ }^{42}$ and/or who have an ISI score below the cut-off of 10 at post-treatment, ${ }^{38}$ relative to $\mathrm{CP}$ and no treatment.

Actigraphy. Objective sleep-wake data will be calculated from actigraphy watches (GENEActive, Activinsights, Cambridgeshire, UK). These are small, wrist-worn accelerometers that record daily movement and can be used to calculate a range of objective sleep parameters. Actigraphy watches have established validity against gold standard sleep assessment (ie, polysomnography.) ${ }^{43}$ Actigraphy data will be used to calculate objective sleep parameters including sleep onset latency, total sleep duration and overall sleep quality.

Consensus sleep diary (CSD). ${ }^{44}$ The CSD is widely used to assess participants' self-reported sleep patterns. The CSD includes questions about time in bed, time to sleep, and number and duration of awakenings. As a measure of treatment adherence, OLP and CP participants will complete items asking whether, and when, they took the capsules the previous night.

Fatigue Symptom Inventory (FSI). ${ }^{45}$ The FSI is a 14 -item self-report inventory assessing the intensity, duration, impact and daily pattern of fatigue over a 1-week period. Participants rate their fatigue from $0=$ 'no fatigue' to $10=$ 'the most fatigue' with respect to severity, duration and interference. Individual items are scored to assess least, most and average fatigue in the past week, and current fatigue. Severity items can be averaged to obtain a composite FSI score. ${ }^{46}$ Items addressing fatigue interference with daily functioning or psychosocial well-being are averaged to obtain an interference scale score. ${ }^{47}$ The FSI has good internal consistency (0.91-0.96), and demonstrated concurrent, convergent and discriminant validity. $^{47}$
Depression Anxiety Stress Scales (DASS-21). ${ }^{48}$ The DASS-21 is a 21-item self-report measure consisting of three 7-item scales measuring symptoms of depression, anxiety and stress. Each item is rated on a scale from $0=$ 'did not apply to me at all' to $4=$ 'applied to me very much, or most of the time'. Item scores are summed and multiplied by two to calculate a final score ranging from 0 to 42 for each scale. Psychometric properties indicate adequate construct validity and excellent internal consistency for the depression (0.88), anxiety (0.82) and stress (0.90) scales. ${ }^{49}$

Expectancy measure. A purpose-built expectancy measure was developed for this study. All participants are asked how much they expect their insomnia symptoms to change as a result of taking part of the study at two time points: prior to the 2-week baseline period and prior to the 2-week treatment period (after randomisation). Responses are completed on a scale from $-10=$ 'much worse' through $0=$ 'no change' to $10=$ 'much better'.

Generic Assessment of Side Effects (GASE). ${ }^{50}$ The GASE is a standardised self-report measure of 36 commonly reported side effects observed in clinical trials (eg, headache, dry mouth). Participants rate the intensity of these symptoms from $0=$ 'not present' to $3=$ 'severe' and indicate whether each symptom is related to their treatment. The intensity ratings are summed to obtain a total GASE score and a medication-attributed score is calculated by summing symptom scores rated as related to treatment. ${ }^{50}$ Because OC does not receive any medication, an amended version of the attribution question will be administered, whereby for any symptoms present participants in all three arms (OLP, CP and OC) indicate whether each symptom is related to study participation first, then only those participants in the OLP and CP arms indicate whether they believe any such symptom is related to the study medication.

Treatment Satisfaction Questionnaire for Medication-Version II $\left(\right.$ TSQM-II). ${ }^{51}$ The TSQM-II is an 11-item self-report measure of participants' perceived effectiveness, convenience, side effects and overall satisfaction with medication use. The measure will be administered specifically to participants enrolled in the OLP or CP arms because it focuses on treatment/medication. Domain items are summed and then transformed to a composite score ranging from 0 to 100 . The TSQM-II has demonstrated construct validity and internal consistency coefficients ranging from 0.88 to 0.94 across domains. ${ }^{51} 52$

\section{Potential predictors of uptake and the placebo effect}

In addition to demographic data, the following personality and clinical history measures will be administered as part of the online screening measures completed prior to study enrolment.

Life Orientation Test-Revised (LOT-R). ${ }^{53}$ The LOT-R is a 10-item measure assessing dispositional optimism. Responses are made on a 5-point scale from $0=$ "strongly disagree' to 4='strongly agree' to items such as 'I'm always optimistic about my future', with six of the items summed to achieve an overall optimism score. Psychometric 
Table 1 Summary of descriptions and discussion points for open-label placebo (OLP) and conventional placebo (CP)

\begin{tabular}{|c|c|c|}
\hline $\begin{array}{l}\text { Discussion } \\
\text { point }\end{array}$ & OLP & CP \\
\hline $\begin{array}{l}\text { What is this } \\
\text { treatment? }\end{array}$ & $\begin{array}{l}\text { Placebo capsules } \\
\text { containing no active } \\
\text { ingredient. }\end{array}$ & $\begin{array}{l}\text { A new } \\
\text { pharmacological } \\
\text { agent, (drug } \\
\text { codename). }\end{array}$ \\
\hline $\begin{array}{l}\text { What does } \\
\text { previous } \\
\text { research } \\
\text { say? }\end{array}$ & $\begin{array}{l}\text { Placebo effects have } \\
\text { been found to reduce } \\
\text { insomnia symptoms, } \\
\text { but deception is } \\
\text { typically involved. Some } \\
\text { recent studies in other } \\
\text { countries have shown } \\
\text { OLP effects outside of } \\
\text { sleep. }\end{array}$ & $\begin{array}{l}\text { Some recent } \\
\text { studies in other } \\
\text { countries have } \\
\text { shown that (drug } \\
\text { codename) can } \\
\text { reduce insomnia } \\
\text { symptoms. }\end{array}$ \\
\hline $\begin{array}{l}\text { What are the } \\
\text { mechanisms } \\
\text { of action? }\end{array}$ & $\begin{array}{l}\text { Placebo effects trigger } \\
\text { the brain to release } \\
\text { neurotransmitters that } \\
\text { can improve symptoms. } \\
\text { These responses can be } \\
\text { automatic. }\end{array}$ & $\begin{array}{l}\text { (Drug codename) } \\
\text { triggers the } \\
\text { brain to release } \\
\text { neurotransmitters } \\
\text { that can improve } \\
\text { sleep. }\end{array}$ \\
\hline $\begin{array}{l}\text { How should } \\
\text { I take the } \\
\text { capsules? }\end{array}$ & \multicolumn{2}{|c|}{$\begin{array}{l}\text { Work best if taken exactly as prescribed. A } \\
\text { positive attitude helps, but is not essential. }\end{array}$} \\
\hline $\begin{array}{l}\text { How long } \\
\text { will it take to } \\
\text { work? }\end{array}$ & \multicolumn{2}{|c|}{$\begin{array}{l}\text { Generally work quickly, but can take longer } \\
\text { for some people. }\end{array}$} \\
\hline
\end{tabular}

properties indicate adequate construct validity and modest internal consistency correlations ranging from 0.43 to $0.63 .^{53}$

Big-Five Inventory (BFI)_openness to experience. ${ }^{54}$ The BFI is a widely used taxonomy of personality traits. Ten selfreport items assessing the domain openness to experience were selected for this trial. The BFI has good construct validity and convergent validity with other similar personality measures. ${ }^{54}$

Insomnia treatment history. A purpose-designed measure was developed to assess participants' self-reported history of treatments for insomnia (pharmacological, psychological, complementary) and their perceived efficacy of these treatments.

\section{Procedure}

Figure 1 shows the study flow. Eligible participants will be invited to attend their first on-site visit (visit 1). At visit 1 , all participants will be given an actigraphy watch to wear and CSD to complete, for the 2-week baseline period. Participants will return to the study site for visit 2 (day 14) and complete outcome measures. At visit 2, they will be randomised to one of three conditions: OLP, CP or OC. In the case of the placebo arms, the researcher will discuss the relevant treatment with each participant according to five points, summarised in table 1, with the OLP information guided by previous OLP trials. ${ }^{19} 20$ In the OLP arm there will be an additional emphasis on explaining that most placebo research in the past has involved deception, while those in the CP arm will be provided with information about the fake medication.

Participants who accept an invite to OLP or CP will be provided with placebo capsules and the dosage instructions, which require them to take two placebo capsules 10-15 min prior to going to bed for the 2-week treatment period. Participants will be asked to record their daily treatment adherence in the CSD. Participants who decline an invite to the OLP or CP arms will continue in the study, unless they choose to withdraw. During the treatment period, all participants will continue completing the CSD and wearing the actigraphy watch. At the final study visit (visit 3), all participants will return the CSD and actigraphy watches, and participants in the OLP and CP arms will return the capsule bottles and any unused capsules as an additional measure of treatment adherence. All participants will complete post-treatment outcome measures and be debriefed at the end of their study participation. On-site study visits may be replaced with video-link visits in the event that COVID-19 social distancing requirements prevent face-to-face interactions, with study materials being couriered if necessary.

\section{Sample size}

There have been no previous studies on OLP for insomnia. Charlesworth et $a l \mathrm{~s}^{28}$ meta-analysis of OLP for other conditions (eg, chronic pain, irritable bowel syndrome) found a large effect size of $d=0.88$ relative to no treatment. Assuming a similar effect size, to obtain $80 \%$ power with alpha $=0.05$, we would require 22 participants to detect this effect size comparing OLP and OC arms. However, we are also seeking to determine whether OLPs differ in efficacy relative to CPs-which has not been investigated systematically. We hypothesise that OLP will be less effective than CP and that the effect size for this comparison will be weaker than the effect size for OLP versus OC. To detect an effect size for OLP versus CP of $d=0.5$, we will require 64 participants per type of placebo treatment to achieve $80 \%$ power with alpha $=0.05$. Therefore, using an allocation ratio of 2:2:1, we would require 64, 64, 32 (total $\mathrm{N}=160$ ) participants for OLP, CP and OC, respectively, to obtain sufficient power for both of the critical comparisons. However, because the cmRCT involves twostage consent process, we will recruit $\mathrm{N}=267$ participants into the initial cohort aiming to randomise 107 to each placebo arm and 53 to OC, which assumes at least twothirds $(67 \%)$ uptake in the placebo arms, including allowance for $10 \%$ attrition. This will provide us with sufficient power for both intent-to-treat (ITT) (primary) and perprotocol (sensitivity) analyses.

\section{Randomisation and blinding}

Randomisation tables will be generated using randomizer.org. Randomisation will be conducted on a 2:2:1 ratio (OLP, CP, OC) and stratified according to gender and scores on the ISI $(<15$ and $\geq 15)$. Randomisation will take place after the eligibility screening and baseline 
assessment (allocation concealment) at visit 2. Blinding of the participant and researcher administering the treatment is not possible, however, data analysis will be performed by a blinded member of the team.

\section{Statistical analysis \\ Primary outcome}

ISI. ITT will be used as the primary analysis to compare the effect of OLP, CP and OC on insomnia symptoms. The primary endpoint (mean scores on the ISI posttreatment) will be assessed using a multilevel model with group (OLP, CP, OC) and baseline (visit 2) ISI score included as factors. Consistent with previous analysis methods in cmRCTs, the ITT population for the OLP and CP arms will consist of all participants who receive an offer of treatment, regardless of treatment uptake. As a secondary sensitivity analysis, a per-protocol approach will also be implemented including only those participants in the OLP, CP and OC arms who complete the study. The analyses will include participants who scored $\geq 10$ on the ISI at screening, but we will also conduct sensitivity analysis excluding any participants who fall below this threshold during the baseline period (assessed at visit 2).

\section{Secondary outcomes}

Uptake. A $\mathrm{X}^{2}$ test of independence will be used to determine whether rates of accepting treatment differ when OLP versus CP is offered.

Response rate. Group differences in the proportion of participants achieving a clinically significant response (ie, $\geq 6$-point reduction and/or $<10$ on the ISI) will be analysed using a $\mathrm{X}^{2}$ test of independence.

Other sleep parameters and outcomes. Other sleep measures (self-report and objective), daytime fatigue, depression, anxiety, stress, treatment satisfaction, expectancy and side effects will be assessed as per the primary ISI outcome.

\section{Predictors of uptake and the placebo effect}

Potential predictors of uptake and the placebo effect will be assessed using a combination of logistic and linear regressions to identify which clinical, demographic and personality characteristics predict uptake (logistic) of and the placebo effect (linear: ISI scores and related outcomes; logistic: response rates) to OLP and CP.

For all analyses, results will be considered statistically significant when $\mathrm{p}<0.05$.

\section{Patient and public involvement}

Neither patients nor members of the public had any involvement in the design of the OPIN trial.

\section{ETHICS AND DISSEMINATION}

The study is registered with the Australian and New Zealand Clinical Trial Registry (see online supplemental material 1). The study protocol (version 6 dated 10 September 2020), participant information sheets and consent forms (see online supplemental material 2) and relevant materials, and the ethical aspects of this trial have been reviewed and approved by The University of Sydney Human Research Ethics Committee (HREC 2019/552). Study data will be collected and stored using the university's Research Electronic Data Capture system, with password-protected access provided to relevant research personnel only. All data will be securely stored for a minimum of 15 years. The PI will be responsible for communicating important protocol modifications. The final dataset will be maintained by the PI, with de-identified participant data available on request following publication to researchers providing a methodologically and ethically sound proposal, in addition to the full study protocol, statistical analysis plan and analytical code. Results from this trial will be disseminated in the form of peer-reviewed conference proceedings and publications.

Contributors $\mathrm{BC}$ conceptualised the study and is the principal investigator and grant holder. BC, AS, LS, NG, DSJC, DB and ZA made significant contributions to designing the study. BC, AS, LS, NG, DB and ZA contributed to developing the screening procedures. $B C$ and DSJC were responsible for the power calculations and statistical analysis plan. $B C, A S$ and $Z A$ were responsible for creating the first draft of this manuscript. BC, AS, LS, NG, DSJC, DB and ZA provided input and feedback, and approved the final draft of this manuscript.

Funding This work was supported by a University of Sydney Psychology Seed Grant 2019 and a University of Sydney Research Accelerator Prize 2020.

Competing interests None declared.

Patient consent for publication Not required.

Provenance and peer review Not commissioned; externally peer reviewed.

Supplemental material This content has been supplied by the author(s). It has not been vetted by BMJ Publishing Group Limited (BMJ) and may not have been peer-reviewed. Any opinions or recommendations discussed are solely those of the author(s) and are not endorsed by BMJ. BMJ disclaims all liability and responsibility arising from any reliance placed on the content. Where the content includes any translated material, BMJ does not warrant the accuracy and reliability of the translations (including but not limited to local regulations, clinical guidelines, terminology, drug names and drug dosages), and is not responsible for any error and/or omissions arising from translation and adaptation or otherwise.

Open access This is an open access article distributed in accordance with the Creative Commons Attribution Non Commercial (CC BY-NC 4.0) license, which permits others to distribute, remix, adapt, build upon this work non-commercially, and license their derivative works on different terms, provided the original work is properly cited, appropriate credit is given, any changes made indicated, and the use is non-commercial. See: http://creativecommons.org/licenses/by-nc/4.0/.

ORCID iD

Ben Colagiuri http://orcid.org/0000-0003-2157-2641

\section{REFERENCES}

1 Roth T, Roehrs T. Insomnia: epidemiology, characteristics, and consequences. Clin Cornerstone 2003;5:5-15.

2 Riemann D, Baglioni C, Bassetti C, et al. European guideline for the diagnosis and treatment of insomnia. J Sleep Res 2017;26:675-700.

3 Ree M, Junge M, Cunnington D. Australasian sleep association position statement regarding the use of psychological/behavioral treatments in the management of insomnia in adults. Sleep Med 2017;36:S43-7.

4 Zhang Y, Ren R, Lei F, et al. Worldwide and regional prevalence rates of co-occurrence of insomnia and insomnia symptoms with obstructive sleep apnea: a systematic review and meta-analysis. Sleep Med Rev 2019;45:1-17.

5 Appleton SL, Gill TK, Lang CJ, et al. Prevalence and comorbidity of sleep conditions in Australian adults: 2016 sleep health foundation national survey. Sleep Health 2018;4:13-19. 
6 Bjorvatn B, Meland E, Flo E, et al. High prevalence of insomnia and hypnotic use in patients visiting their general practitioner. Fam Pract 2017;34:20-4.

7 Charles J, Harrison C, Britt H. Insomnia. Aust Fam Physician 2009;38:283-83.

8 Miller CB, Valenti L, Harrison CM, et al. Time trends in the family physician management of insomnia: the Australian experience (20002015). J Clin Sleep Med 2017;13:785-90.

9 American Psychiatric Association. Diagnostic and statistical manual of mental disorders. Arlington: American Psychiatric Publishing, 2013.

10 Mai E, Buysse DJ. Insomnia: prevalence, impact, pathogenesis, differential diagnosis, and evaluation. Sleep Med Clin 2008;3:167-74.

11 Sofi F, Cesari F, Casini A, et al. Insomnia and risk of cardiovascular disease: a meta-analysis. Eur J Prev Cardiol 2014;21:57-64.

12 Van Cauter E. Sleep disturbances and insulin resistance. Diabet Med 2011;28:1455-62.

13 Hertenstein E, Feige B, Gmeiner T, et al. Insomnia as a predictor of mental disorders: a systematic review and meta-analysis. Sleep Med Rev 2019;43:96-105.

14 Sateia MJ, Buysse DJ, Krystal AD, et al. Clinical practice guideline for the pharmacologic treatment of chronic insomnia in adults: an American academy of sleep medicine clinical practice guideline. $J$ Clin Sleep Med 2017;13:307-49.

15 Buscemi N, Vandermeer B, Hooton N, et al. The efficacy and safety of exogenous melatonin for primary sleep disorders. A meta-analysis. $J$ Gen Intern Med 2005;20:1151-8.

16 Ogeil RP, Chakraborty SP, Young AC, et al. Clinician and patient barriers to the recognition of insomnia in family practice: a narrative summary of reported literature analysed using the theoretical domains framework. BMC Fam Pract 2020;21:1-10.

17 Adams R, Appleton S, Taylor A. Report to the sleep health foundation 2016 sleep health survey of Australian adults, 2016. Available: https://www sleephealthfoun dation org au/pdfs/surveys/ SleepHealthFoundation-Survey pdf

18 Bélanger $L$, Vallières $A$, Ivers $\mathrm{H}$, et al. Meta-analysis of sleep changes in control groups of insomnia treatment trials. J Sleep Res 2007;16:77-84.

19 Winkler A, Rief W. Effect of placebo conditions on polysomnographic parameters in primary insomnia: a meta-analysis. Sleep 2015;38:925-31.

20 McCall WV, D'Agostino R, Dunn A. A meta-analysis of sleep changes associated with placebo in hypnotic clinical trials. Sleep Med 2003;4:57-62.

21 Colagiuri B, Schenk LA, Kessler MD, et al. The placebo effect: from concepts to genes. Neuroscience 2015;307:171-90.

22 Kaptchuk TJ, Miller FG. Placebo effects in medicine. N Engl J Med 2015;373:8-9.

23 Enck P, Bingel U, Schedlowski M, et al. The placebo response in medicine: minimize, maximize or personalize? Nat Rev Drug Discov 2013;12:191-204.

24 Yeung V, Sharpe L, Glozier N, et al. A systematic review and metaanalysis of placebo versus no treatment for insomnia symptoms. Sleep Med Rev 2018;38:17-27.

25 Barnett AG, van der Pols JC, Dobson AJ. Regression to the mean: what it is and how to deal with it. Int J Epidemiol 2005;34:215-20.

26 Rutherford BR, Roose SP. A model of placebo response in antidepressant clinical trials. Am J Psychiatry 2013;170:723-33.

27 Biller-Andorno N. The use of the placebo effect in clinical medicineethical blunder or ethical imperative? Sci Eng Ethics 2004;10:43-50.

28 Charlesworth JEG, Petkovic G, Kelley JM, et al. Effects of placebos without deception compared with no treatment: a systematic review and meta-analysis. J Evid Based Med 2017;10:97-107.

29 Carvalho C, Caetano JM, Cunha L, et al. Open-label placebo treatment in chronic low back pain: a randomized controlled trial. Pain 2016;157:2766-72.

30 Kaptchuk TJ, Friedlander E, Kelley JM, et al. Placebos without deception: a randomized controlled trial in irritable bowel syndrome. PLoS One 2010:5:e15591.

31 Kelley JM, Kaptchuk TJ, Cusin C, et al. Open-Label placebo for major depressive disorder: a pilot randomized controlled trial. Psychother Psychosom 2012;81:312-4.

32 Schaefer M, Sahin T, Berstecher B. Why do open-label placebos work? A randomized controlled trial of an open-label placebo induction with and without extended information about the placebo effect in allergic rhinitis. PLoS One 2018;13:e0192758.

33 Evers AWM, Colloca L, Blease C, et al. Implications of placebo and nocebo effects for clinical practice: expert consensus. Psychother Psychosom 2018;87:204-10.

34 Blease CR, Bernstein MH, Locher C. Open-label placebo clinical trials: is it the rationale the interaction or the pill? BMJ Evid Based Med 2020;25:159-65.

35 Relton C, Torgerson D, O'Cathain A, et al. Rethinking pragmatic randomised controlled trials: introducing the "cohort multiple randomised controlled trial" design. BMJ 2010;340:c1066.

36 Chan A-W, Tetzlaff JM, Altman DG, et al. SPIRIT 2013 statement: defining standard protocol items for clinical trials. Ann Intern Med 2013;158:200-7.

37 Bastien $\mathrm{CH}$, Vallières $\mathrm{A}$, Morin $\mathrm{CM}$. Validation of the insomnia severity index as an outcome measure for insomnia research. Sleep Med 2001;2:297-307.

38 Morin CM, Belleville G, Bélanger L, et al. The insomnia severity index: psychometric indicators to detect insomnia cases and evaluate treatment response. Sleep 2011;34:601-8.

39 Hartescu I, Morgan K, Stevinson CD. Increased physical activity improves sleep and mood outcomes in inactive people with insomnia: a randomized controlled trial. J Sleep Res 2015;24:526-34.

40 Herring WJ, Connor KM, Ivgy-May N, et al. Suvorexant in patients with insomnia: results from two 3-month randomized controlled clinical trials. Biol Psychiatry 2016;79:136-48.

41 Espie CA, Kyle SD, Williams C, et al. A randomized, placebocontrolled trial of online cognitive behavioral therapy for chronic insomnia disorder delivered via an automated media-rich web application. Sleep 2012;35:769-81.

42 Yang M, Morin CM, Schaefer K, et al. Interpreting score differences in the insomnia severity index: using health-related outcomes to define the minimally important difference. Curr Med Res Opin 2009;25:2487-94.

43 Lichstein KL, Stone KC, Donaldson J, et al. Actigraphy validation with insomnia. Sleep 2006;29:232-41.

44 Carney CE, Buysse DJ, Ancoli-Israel S, et al. The consensus sleep diary: standardizing prospective sleep self-monitoring. Sleep 2012;35:287-302.

45 Hann DM, Jacobsen PB, Azzarello LM, et al. Measurement of fatigue in cancer patients: development and validation of the fatigue symptom inventory. Qual Life Res 1998;7:301-10.

46 Donovan KA, Jacobsen PB, Small BJ, et al. Identifying clinically meaningful fatigue with the fatigue symptom inventory. $J$ Pain Symptom Manage 2008;36:480-7.

47 Donovan KA, Jacobsen PB. The fatigue symptom inventory: a systematic review of its psychometric properties. Supportive Care in Cancer 2011;19:169-85.

48 Lovibond PF, Lovibond SH. The structure of negative emotional states: comparison of the depression anxiety stress scales (DASS) with the Beck depression and anxiety inventories. Behav Res Ther 1995;33:335-43.

49 Henry JD, Crawford JR. The short-form version of the depression anxiety stress scales (DASS-21): construct validity and normative data in a large non-clinical sample. Br J Clin Psychol 2005;44:227-39.

50 Rief W, Glombiewski J, Barsky A. Generic assessment of side effects: GASE. Verlag, Bern: Hans Huber, 2009.

51 Atkinson MJ, Sinha A, Hass SL, et al. Validation of a general measure of treatment satisfaction, the treatment satisfaction questionnaire for medication (TSQM), using a national panel study of chronic disease. Health Qual Life Outcomes 2004;2:12.

52 Atkinson MJ, Kumar R, Cappelleri JC, et al. Hierarchical construct validity of the treatment satisfaction questionnaire for medication (TSQM version II) among outpatient pharmacy consumers. Value Health 2005;8:S9-24.

53 Scheier MF, Carver CS, Bridges MW. Distinguishing optimism from neuroticism (and trait anxiety, self-mastery, and self-esteem): a reevaluation of the life orientation test. J Pers Soc Psychol 1994;67:1063-78.

54 John OP, Srivastava S. The big five trait taxonomy: history, measurement, and theoretical perspectives. Handbook Personal: Theory Res 1999;2:102-38. 